LBNL-55463

August 2004

\title{
ON THE SPEED OF GRAVITY AND THE JUPITER/QUASAR MEASUREMENT
}

\author{
STUART SAMUEL ${ }^{1}$ \\ Theory Group, MS 50A-5101 \\ Lawrence Berkeley National Laboratory \\ One Cyclotron Road \\ Berkeley, CA 94720 USA \\ ${ }^{1}$ samuel@thsrv.lbl.gov
}

\begin{abstract}
I present the theory and analysis behind the experiment by Fomalont and Kopeikin involving Jupiter and quasar J0842+1845 that purported to measure the speed of gravity. The computation of the $v_{J} / c$ correction to the gravitational time delay difference relevant to the experiment is derived, where $v_{J}$ is the speed of Jupiter as measured from Earth. Since the $v_{J} / c$ corrections are too small to have been measured in the Jupiter/quasar experiment, it is impossible that the speed of gravity was extracted from the data, and I explain what when wrong with the data analysis. Finally, mistakes are shown in papers by Fomalont and Kopeikin intended to rebut my work and the work of others.
\end{abstract}

Keywords: speed of gravity,general relativity,quasar J0842+1845

PACS indices: $04.20 . \mathrm{Cv}, 04.80 . \mathrm{Cc}, 04.25 . \mathrm{Nx}, 98.54 . \mathrm{Aj}, 96.30 . \mathrm{Kf}$ 


\section{Introduction}

Albert Einstein constructed his general theory of relativity so that classical gravity would be compatible with the principles of special relativity. As such, gravitational waves and the influences of gravity are suppose to propagate at the speed of light $c$. For example, if hypothetically the Sun were to explode into two pieces then the force of gravity on the Earth would change. However, it would not happen suddenly but 8 and $1 / 3$ minutes later, since this is the time it takes gravitational effects (and light) to travel from the Sun to the Earth. In other words, the Earth would continue in its almost circular orbit as governed by the gravity of a single massive central body for another 8 and $1 / 3$ minutes, only after which would its motion be determined by the two exploding pieces. Since gravitational waves have not yet been detected, it has not been possible to test whether they travel at the speed $c$, nor has there been a system in which the speed of propagation of gravitational influences $c_{g}$ has been measured.

The lack of a measurement of $c_{g}$ inspired S. Kopeikin to propose an experiment to test whether $c_{g}$ is the speed of light.[1] The ideas behind his proposal are outlined in the next four paragraphs.

When electromagnetic waves pass by a massive object $M$, two effects occur: Firstly, the waves are very slighly bent, and, secondly, there is a tiny delay in the transmission time. Both are prominent effects of general relativity, and the latter is known as the Shapiro time delay. $[2,3,4]$

The physical solution of Einstein's equations involve the position $\vec{x}_{M}$ of gravitygenerating objects at retarded times:

$$
t_{r e t}=t-\left|\vec{x}-\vec{x}_{M}\left(t_{r e t}\right)\right| / c .
$$

Here, $\vec{x}$ is the location at which gravity is exerting its influence. The use of retarded times $t_{r e t}$ as given in Eq. (1.1) implies that the effect of gravity propagates at $c$. To allow for gravity to propagate at a different speed $c_{g}$, one would expect to replace Eq. (1.1) by

$$
t_{r e t}=t-\left|\vec{x}-\vec{x}_{M}\left(t_{r e t}\right)\right| / c_{g} .
$$

On September 8, 2002, a conjunction of Jupiter and quasar J0842+1845 took place. Kopeikin argued that this event could be used to measure the speed of prop- 
agation of gravity.[1] The thinking behind his proposal originates from the previous paragraph. Being very far away, the location of quasar J0842+1845 in the sky is virtually fixed. Jupiter, however, moves. Its position at the retarded time depends sensitively on its velocity and on $c_{g}$ through Eq. (1.2). Therefore, one might expect that a precise measurement of the Shapiro time delay due to Jupiter on the signal from quasar J0842+1845 would permit a determination of $c_{g}$.

The possible effect of Jupiter's velocity $\vec{v}_{J}$ on the Shapiro time delay can be illustrated from an example: Suppose that Jupiter is moving toward the direction of the quasar waves. If $c_{g}$ were infinite, which corresponds to the Newtonian limit of general relativity, then it would appear that the instantaneous position of Jupiter would be relevant. If $c_{g}=c$, then the position of Jupiter evaluated at the retarded time is somewhat farther away than the instantaneous position, and if $c_{g}<c$, then the position as determined by Eq. (1.2) is even further away. See Figure 1.

Using an array of radio telescopes that stretched across the United States all the way to Germany, E. Fomalont and S. Kopeikin measured the tiny Shapiro time delays. By applying Very Long Baseline Interferometry (VLBI), they achieved a remarkable sensitivity at the picosecond level. The results for $c_{g}$, which were announced in January 2003 meeting of the American Astronomical Society held in Seattle, Washington, immediately caught the attention of the media. The New York Times, for example, featured an article entitled "Einstein Was Right on Gravity's Speed." [5] At the meeting, Fomalont and Kopeikin announced that $c_{g}=c$ to within $20 \%$ and this result was subsequently published.[6]

If this result is correct then it is a fundamental confirmation of Einstein's general theory of relativity. However, shortly after the American Astronomical Society meeting, a debate among astrophysicists arose about the Jupiter/quasar experiment. Several papers appeared arguing that Fomalont and Kopeikin had not accomplished their goal of measuring $c_{g}$. H. Asada had published an early work[7] stating that Kopeikin's idea actually measures the speed of light instead of the speed of gravity, C. M. Will also argued that the measurements were not directly sensitive to $c_{g},[8]$ and other papers also appeared criticizing the theory behind the experiment. $[9,10,11]$ The difficulty that many of the above works were addressing is that there is no agreed 
upon method for extending Einstein's theory to the case for which $c_{g} \neq c$. Kopeikin has repeatly tried to defend these criticisms of his work.[12, 13]

In a Physical Review Letter[14] (hereafter referred to as my Physical Review Letter), I bypassed the issue of how to extend general relativity to the case where the speed of gravity does not equal the speed of light and computed the $v_{J} / c$ corrections to the Shapiro time delay in Einstein's theory using a relatively simple method. The $v_{J} / c$ corrections did not agree with those derived by Kopeikin[1, 12] when his parameter $c_{g}$ was set to $c$. The correct theoretical formula implied that the $v_{J} / c$ dependence was at least 100 times smaller than could have been measured by the array of radio telescopes used in the Fomalont/Kopeikin experiment. In other words, the speed of gravity could not have been extracted from the Jupiter/quasar measurement. My Physical Review Letter definitively settled the speed of gravity controversy: The parameter $c_{g}$ has not been measured.

Kopeikin had argued that there is an enhancement in the $v_{J} / c$ correction to the Shapiro time delay by a factor of $1 / \theta$ where $\theta$ is the angle between Jupiter and the quasar. $[1,12]$ Since $\theta$ is small, it would seem to be that the $v_{J} / c$ correction is sizeable. However, no such $1 / \theta$ enhancement is actually present.

My Physical Review Letter pinpointed the source of the discrepancy. The leadingorder, velocity-independent part of the term that Fomalont and Kopeikin measured depends on the distance $\xi$ of closest approach of the radios waves to Jupiter as $1 / \xi$. This distance is determined by the position of Jupiter and the radio waves as the latter pass by Jupiter. If $R_{E J}$ and $\theta_{o b s}$ respectively denote the Earth-Jupiter distance and the angle that an astronomer observes between Jupiter and the quasar, then

$$
\xi=R_{E J} \theta_{o b s}
$$

The angle $\theta_{o b s}$ is the one determined by the geometry of the positions of Jupiter, the quasar and the Earth at the time in which the radio waves pass by Jupiter.

Fomalont and Kopeikin parametrized their data in terms of an angle $\theta_{1}$ determined by the geometry of the positions of the above three objects at the time in which the radio waves arrived on Earth. These two angles differ: During the time in which the quasar signals travel from Jupiter to Earth, Jupiter moves a significant distance. The 
relation between the two angles is

$$
\theta_{o b s} \approx \theta_{1}+\frac{\vec{n} \cdot \vec{v}_{J}}{c}
$$

where $\vec{n}$ is a unit vector pointing from Jupiter to the quasar's radio waves at the time of closest approach. When the factor $1 / \xi$ appearing in the leading velocity-independent term is expressed in terms of $\theta_{1}$, it appears to become velocity dependent because

$$
\frac{1}{\xi}=\frac{1}{R_{E J} \theta_{o b s}} \approx \frac{1}{R_{E J} \theta_{1}}\left(1-\frac{\vec{n} \cdot \vec{v}_{J}}{c \theta_{1}}\right) .
$$

In summary, parametrizing the Shapiro time delay using $\theta_{1}$ makes the leading term seem to depend on the velocity of Jupiter. Furthermore, the fictitious velocitydependent term appears enhanced.

Fomalont and Kopeikin took their data and fit them to the leading order term but parametrized them in terms of $\theta_{1}$. They then extracted the $v_{J} / c$ dependence calling it the $v_{J} / c_{g}$ correction. Given this procedure and that the data have error bars, it is not surprising that such a procedure produced the purported result that $c_{g}=c$ to within 20\%. The conclusion that the speed of gravity is the speed of light to within experimental errors is not valid due to faulty analysis and a flaw in the theoretical understanding of the situation.

Since the difference between $\theta_{o b s}$ and $\theta_{1}$ is due to the change in the position of Jupiter as the quasar's radio waves propagate from Jupiter to Earth, it is clear that the parameter $c$ in Eq. (1.5) is the speed of light and has nothing to do with the speed of gravity. Therefore there was no justification in using $c_{g}$ in lieu of $c$ when expressing the leading term in terms of $\theta_{1}$.

The measurement of Fomalont and Kopeikin of the Shapiro time delay due to Jupiter is a remarkable experimental achievement. One should remember that the non-Newtonian effects of general relativity due to a planet had hitherto never been detected. However, the experiment has little theoretical significance or fundamental importance, and it indicates nothing about the speed of gravity.

Most of our notation conforms to that of references [1], [12] and [14]. There are several small dimensionless parameters characterizing the Jupiter/quasar measurement: $G_{N} M_{J} /\left(\xi c^{2}\right) \approx 6 \times 10^{-9}$, where $G_{N}$ and $M_{J}$ are respectively Newton's constant and 
the mass of Jupiter; $v_{J} / c \sim 10^{-4} ; B / \xi \leq 0.006$ where $B$ is the distance between any two VLBI stations on Earth; and $\theta_{o b s}=\xi / R_{E J} \sim 0.001$. These parameters, in the order given above, respectively represent the weakness of Jupiter's gravity on the radio waves, the non-relativistic nature of Jupiter's motion, the diminutive size of Earth compared to Jupiter, and the small observational angle between Jupiter and the quasar.

In our analysis, we neglect the square of any of the above quantities. In particular, relativistic and high-order gravitational effects are ignored.

\section{The Leading Order Result}

Suppose that Jupiter is not moving. We refer to this as the static situation. Then, the Shapiro time delay for an electromagnetic wave travelling from the quasar past Jupiter to Earth is

$$
\Delta t=\frac{2 G_{N} M_{J}}{c^{3}}\left(1+\ln \left(\frac{4 R_{J Q} R_{E J}}{\xi^{2}}\right)\right),
$$

where $R_{J Q}$ is the distance from Jupiter to the quasar. Eq. (2.1) is a textbook result.[15]

In the quasar/Jupiter experiment, a series of radio telescopes detected the quasar signals during the conjunction. The time difference $\Delta\left(t_{1}, t_{2}\right)$ between two such Shapiro delays $\Delta t_{2}$ and $\Delta t_{1}$ was measured:

$$
\Delta\left(t_{1}, t_{2}\right)=\Delta t_{2}-\Delta t_{1}
$$

The experimental situation is shown in Figure 2. Signals 1 and 2 propagate from the quasar past Jupiter and arrive at times $t_{1}$ and $t_{2}$ on Earth at detectors located at positions $\vec{x}_{1}\left(t_{1}\right)$ and $\vec{x}_{2}\left(t_{2}\right)$.

Using Eq. (2.1) in Eq. (2.2) yields

$$
\Delta\left(t_{1}, t_{2}\right)=\Delta t_{2}-\Delta t_{1}=\frac{2 G_{N} M_{J}}{c^{3}} \ln \left(\frac{r_{2 J} \xi_{1}^{2}}{r_{1 J} \xi_{2}^{2}}\right) \approx \frac{4 G_{N} M_{J} \Delta \xi}{\xi c^{3}}
$$

where $\Delta \xi=\xi_{1}-\xi_{2}$ and $r_{1 J}$ (respectively, $r_{2 J}$ ) is the distance between the first (respectively, second) detector and Jupiter. The last equality in Eq. (2.3) follows because $\Delta \xi$ is significantly smaller than either $\xi_{1}$ or $\xi_{2}$, and the differences in the 
distances $r_{1 J}$ and $r_{2 J}$ between Jupiter and detector can be neglected. We use $\xi$ without a subscript to denote either of the detector-specific impact parameters $\xi_{1}$ or $\xi_{2}$ when the distinction between the two is not important. Although gravitational effects are often long-ranged, the Shapiro time delay difference $\Delta\left(t_{1}, t_{2}\right)$ is generated in the vicinity of Jupiter as is evident from Eq. (2.3): $\Delta\left(t_{1}, t_{2}\right)$ depends only on impact parameters.

It is convenient to express $\Delta \xi=\xi_{1}-\xi_{2}$ in terms of the displacement between the two detectors $\vec{B}=\vec{x}_{2}\left(t_{2}\right)-\vec{x}_{1}\left(t_{1}\right)$ because $\vec{B}$ is easily determined experimentally. See Figure 2. The radio signals from the quasar are bent slightly by an amount $\Delta \varphi$ as they pass by Jupiter. However, it turns out that this effect can be neglected as we now show.

The bending of a single wave is given by[15]

$$
\Delta \varphi=\frac{4 G_{N} M_{J}}{\xi c^{2}}
$$

The angle that eventually arises between the two rays is

$$
\delta \Delta \varphi=\Delta \varphi_{2}-\Delta \varphi_{1}=\frac{4 G_{N} M_{J} \Delta \xi}{\xi^{2} c^{2}} .
$$

Since the separation between the rays starts as $\Delta \xi$ and increases as the distance times $\delta \Delta \varphi$,

$$
-\vec{n} \cdot \vec{B}=\Delta \xi+R_{E J} \delta \Delta \varphi=\Delta \xi\left(1+\frac{4 G_{N} M_{J} R_{E J}}{\xi^{2} c^{2}}\right) \approx \Delta \xi .
$$

The last equality follows because

$$
\frac{4 G_{N} M_{J} R_{E J}}{\xi^{2} c^{2}} \leq \frac{4 G_{N} M_{J} R_{E J}}{R_{J}^{2} c^{2}} \sim 0.001
$$

where $R_{J}$ is the radius of Jupiter. Since the angular deflection caused by Jupiter is so small, the separation between the two rays remains essentially constant. Indeed, including the second term in Eq. (2.6) in our analysis below only leads to corrections proportional to Newton's constant squared.

Substituting Eq. (2.6) into (2.3), one obtains the following for the static situation

$$
\Delta\left(t_{1}, t_{2}\right)=-\frac{4 G_{N} M_{J} \vec{n} \cdot \vec{B}}{\theta_{o b s} R_{E J} c^{3}}
$$


where we have used $\xi=\theta_{\text {obs }} R_{E J}$. Eq. (2.7) is expressed in quantities measurable on Earth.

\section{The $v_{J} / c$ Corrections}

Now consider the case in which Jupiter is moving with a velocity $\vec{v}_{J}$ with respect to the Earth. In principle, one should start with Einstein's equation

$$
R^{\mu \nu}-\frac{1}{4} g^{\mu \nu} R=-\frac{8 \pi G_{N}}{c^{2}} T^{\mu \nu}
$$

in which $R_{\mu \nu}$, the curvature tensor, is related to the stress-energy tensor $T^{\mu \nu}$.

For a static Jupiter, the stress-energy tensor only has a " 00 " component: $T^{00}=$ $\rho_{J}(x)$, where $\rho_{J}$ is Jupiter's mass density. The metric $g_{\mu \nu}$ in spherical-like coordinates about the center of Jupiter is given by the Schwarzschild solution

$$
c^{2} d \tau^{2}=\left(1-\frac{2 M_{J} G_{N}}{r c^{2}}\right) c^{2} d t^{2}-\left(1-\frac{2 M_{J} G_{N}}{r c^{2}}\right)^{-1} d r^{2}-r^{2} \theta^{2}-r^{2} \sin ^{2} \theta d \varphi^{2},
$$

a result that is only valid exterior to Jupiter.

For a moving Jupiter, the stress-energy tensor has additional components $T^{0 i}$, which are proportional to $v_{J}^{i} / c$ and generate $v_{J} / c$ corrections, and $T^{i j}$, which are proportional to $v_{J}^{i} v_{J}^{j} / c^{2}$ and may be neglected because Jupiter's speed is considerably less than the speed of light. The easiest way to obtain $T^{\mu \nu}, R_{\mu \nu}$ and $g_{\mu \nu}$ for the non-static case is to construct the Lorentz transformation that takes a non-moving Jupiter and sends it moving with velocity $\vec{v}_{J}$. This Lorentz tranformation is then applied to the tensors of the static case. Having obtained the metric for a non-static Jupiter, one would then need to compute the Shapiro time delay for this case.

The above procedure for computing the $v_{J} / c$ corrections to the Shapiro time delay difference $\Delta\left(t_{1}, t_{2}\right)$ is, in principle, the one adopted by Kopeikin and is quite involved.[16] However, some simplifications occur because Jupiter's gravity is weak and $v_{J} / c$ is small. One begins with the Newtonian approximation to Einstein's equations and incorporates the $v_{J} / c$ effects as perturbative corrections, which is the commonly used post-Newtonian approximation.

There is a simpler way to proceed, however, and that is to adopt a reference frame in which Jupiter is static. In such a frame, the Earth moves at a velocity $\vec{v}_{E}$ given by

$$
\vec{v}_{E}=-\vec{v}_{J}
$$


while Jupiter's velocity is zero. The formula in Eq. (2.7) for the static case is then valid. One only needs to incorporate the effects of having moving observation stations on Earth into the Shapiro time delay difference. This procedure is valid because (1) Einstein's theory is Lorentz invariant and (2) during the time in which the quasar rays propagate from Jupiter to the Earth, Jupiter moves almost in a straight line with constant speed. The propagation period is sufficiently short that the orbital motion of Jupiter around the Sun is not important. Since the same is true for the Earth, observers on both planets are essentially inertial during the time scales relevant to the experiment.

Because the Earth is moving, the distance $\vec{B}_{s f}$ between points 1 and 2 as measured in this static-Jupiter frame is not equal to $\vec{B}$ as measured on Earth. In other words, if the first quasar signal arrives at $\vec{x}_{1}\left(t_{1}\right)$ at time $t_{1}$, then the Earth will move a short distance during the time in which it takes the second signal to arrive at $\vec{x}_{2}\left(t_{2}\right)$. Place two observers in the static-frame (meaning that they are not moving with respect to Jupiter) so that one is located at the point 1 at time $t_{1}$ and the another is at the point 2 at time $t_{2}$. Then use these observers to make the time measurements. Since the situation is completely static, the formula for the static case may be used.

The difference between the times at which the two measurements are made is

$$
t_{2}-t_{1}=\left|\vec{x}_{2}\left(t_{2}\right)-\vec{x}_{0}\right| / c-\left|\vec{x}_{1}\left(t_{1}\right)-\vec{x}_{0}\right| / c+\Delta\left(t_{1}, t_{2}\right) .
$$

Here, $\vec{x}_{0}$ is the position of the quasar, and $\left|\vec{x}_{2}\left(t_{2}\right)-\vec{x}_{0}\right| / c-\left|\vec{x}_{1}\left(t_{1}\right)-\vec{x}_{0}\right| / c$ is the time difference that occurs when gravitational effects are absent. Eq. (3.4) is an alternative definition of $\Delta\left(t_{1}, t_{2}\right)$.

The leading contribution to this time difference is

$$
t_{2}-t_{1} \approx-\frac{\vec{K} \cdot \vec{B}}{c}+\Delta\left(t_{1}, t_{2}\right)
$$

where the first term is, in general, larger than the second and arises from the first two terms in Eq. (3.4). Here, $\vec{K}$, which is perpendicular to $\vec{n}$, is a unit vector pointing in the direction of the quasar as seen from Earth. Since during the time $t_{2}-t_{1}$, the Earth moves a distance $\vec{v}_{E}\left(t_{2}-t_{1}\right)$, the displacement between detectors in the static 
frame $\vec{B}_{s f}$ is not the same as that in the Jupiter-moving frame $\vec{B}$ but the two are related by

$$
\vec{B}_{s f}=\vec{B}+\vec{v}_{E}\left(t_{2}-t_{1}\right) \approx \vec{B}-\frac{\vec{K} \cdot \vec{B}}{c} \vec{v}_{E}+\Delta\left(t_{1}, t_{2}\right) \vec{v}_{E}
$$

The Earth's motion gives rise to three $v_{J} / c$ effects. The first occurs because $\vec{B}_{s f}$ needs to be used in Eq. (2.7). When this substitution is performed, a correction of $4 G_{N} M_{J} \vec{n}_{s f} \cdot \vec{v}_{E} \vec{K} \cdot \vec{B} /\left(\xi c^{4}\right)$ is generated. The effect of the last term in Eq. (3.6) may dropped since it is proportional to $G_{N}^{2}$.

The second $v_{J} / c$ correction occurs if the Earth has any motion toward (or away from) Jupiter. For example, if the Earth were moving toward Jupiter, then station 2 would be moving toward the quasar signal 2 during the time after station 1 had detected signal 1 but before station 2 had received signal 2. Station 2 would then record a smaller time delay than if the Earth had not been moving. In other words, the time delay is reduced (or increased) by an amount $\delta \Delta\left(t_{1}, t_{2}\right)$ that is equal to the time it takes light to travel the distance determined by the difference between $\vec{B}_{s f}$ and $\vec{B}$. The corresponding correction due to the second term in Eq. (3.6) is independent of Newton's constant and is a contribution to the first part of Eq. (3.4) that involves detector distance differences. The third term in Eq. (3.6) leads to

$$
\delta \Delta\left(t_{1}, t_{2}\right)=-\frac{\vec{K} \cdot \vec{v}_{E}}{c} \Delta\left(t_{1}, t_{2}\right)
$$

To convert the result to the Jupiter-moving frame, one substitutes $\vec{v}_{E}=-\vec{v}_{J}$. Combining the above two effects with the leading term, one finds[14]

$$
\Delta\left(t_{1}, t_{2}\right)=-\frac{4 G_{N} M_{J}}{\xi c^{3}}\left(\vec{n}_{s f} \cdot \vec{B}\left(1+\frac{\vec{K} \cdot \vec{v}_{J}}{c}\right)+\frac{\vec{K} \cdot \vec{B} \vec{n}_{s f} \cdot \vec{v}_{J}}{c}\right) .
$$

The above result is written in terms of quantities as measured by an observer on Earth with the exception of $\vec{n}_{s f}$, which gives rise to a third effect. Because the Earth is moving with respect to the static frame, the direction of the quasar as observed in the two frames differ: $\vec{K}_{s f} \approx \vec{K}+\left(\vec{n} \cdot \vec{v}_{j} / c\right) \vec{n}$. Since $\vec{n}$ is defined to be perpendicular to $\vec{K}$, it too differs in the two frames: $\vec{n}_{s f} \approx \vec{n}-\left(\vec{n} \cdot \vec{v}_{j} / c\right) \vec{K}$. When $\vec{n}_{s f}$ is substituted into Eq. (3.8), the last term is cancelled:

$$
\Delta\left(t_{1}, t_{2}\right)=-\frac{4 G_{N} M_{J}}{\xi c^{3}} \vec{n} \cdot \vec{B}\left(1+\frac{\vec{K} \cdot \vec{v}_{J}}{c}\right)
$$


As indicated by a lack of subscripts $s f$, all quantities are now those measured by an observer on Earth. Equation (3.9) has no 1/ $\theta_{\text {obs }}^{2}$ term so that there is no enhancement of the velocity-dependent effects.

The leading term for $\Delta\left(t_{1}, t_{2}\right)$ in Eq. (3.9) is of order $10^{-10}$ seconds, well within the measuring capability of the Jupiter/quasar experiment. However, the $v_{J} / c$ term is not bigger than $10^{-14}$ seconds, which is more than 100 times smaller than what was measurable by the VLBI stations. Therefore reference [6] was insensitive to the $v_{J} / c$ term. Furthermore, this $v_{J} / c$ correction is masked by larger corrections that are suppressed by factors such as $B / \xi$ and $\theta_{\text {obs }}$ compared to the leading term. The above analysis leaves no doubt that Fomalont and Kopeikin did not measure the speed of gravity.

Here is an example of a correction down by the order of $\theta_{\text {obs }}$ that can be up to five times bigger than the $v_{J} / c$ term. If $\vec{B}$ has a component in the direction of the quasar, then the differences in the distances $r_{1 J}$ and $r_{2 J}$ in Eq. (2.3) generate the following correction:

$$
\frac{2 G_{N} M_{J}}{c^{3}} \frac{\vec{K} \cdot \vec{B}}{R_{E J}}
$$

It is smaller than the leading term by a factor of $0.5 \xi / R_{E J} \sim 0.5 \theta_{\text {obs }}$.

\section{The Kopeikin Formula for $\Delta\left(t_{1}, t_{2}\right)$}

Using the post-Newtonian approximation, Kopeikin obtained the following result for $\Delta\left(t_{1}, t_{2}\right)[12]$

$$
\Delta\left(t_{1}, t_{2}\right)=\left(1+\frac{\vec{K} \cdot \vec{v}_{J}}{c}\right) \frac{2 G_{N} M_{J}}{c^{3}} \ln \left[\frac{r_{1 J}\left(s_{1}\right)+\vec{K} \cdot \vec{r}_{1 J}\left(s_{1}\right)}{r_{2 J}\left(s_{2}\right)+\vec{K} \cdot \vec{r}_{2 J}\left(s_{2}\right)}\right],
$$

where $\vec{r}_{1 J}\left(s_{1}\right) \equiv \vec{x}_{1}-\vec{x}_{J}\left(s_{1}\right)$ and $\vec{r}_{2 J}\left(s_{2}\right) \equiv \vec{x}_{2}-\vec{x}_{J}\left(s_{2}\right)$ are respectively the distance vectors between the observation points 1 and 2 and Jupiter evaluated at the retarded times

$$
\begin{aligned}
& s_{1}=t_{1}-\left|\vec{x}_{1}-\vec{x}_{J}\left(s_{1}\right)\right| / c, \\
& s_{2}=t_{2}-\left|\vec{x}_{2}-\vec{x}_{J}\left(s_{2}\right)\right| / c .
\end{aligned}
$$

Note that Kopeikin generally works in a frame in which Jupiter is moving and the Earth is not so that Jupiter's position $\vec{x}_{J}(t)$ varies with time $t$, while that of a detector station does not. 
The term in Eq. (4.1) involving $\vec{K} \cdot \vec{v}_{J} / c$ is discarded by Fomalont and Kopeikin due to its smallness. It is the main $v_{J} / c$ correction in Eq. (3.9).

At time $s$, let $\theta_{1}(s)$ (respectively, $\theta_{2}(s)$ ) be the angle between $\vec{r}_{1 J}(s)$ (respectively, $\left.\vec{r}_{1 J}(s)\right)$ and $\vec{K}$ (the unit vector pointing toward the quasar). Then

$$
\begin{aligned}
& r_{1 J}(s)+\vec{K} \cdot \vec{r}_{1 J}(s)=r_{1 J} \theta_{1}^{2}(s) / 2+O\left(\theta_{1}^{4}(s)\right), \\
& r_{2 J}(s)+\vec{K} \cdot \vec{r}_{2 J}(s)=r_{2 J} \theta_{2}^{2}(s) / 2+O\left(\theta_{2}^{4}(s)\right) .
\end{aligned}
$$

The times $s_{1}$ and $s_{2}$ at which one is to evaluate $\vec{r}_{1 J}\left(s_{1}\right)$ and $\vec{r}_{2 J}\left(s_{2}\right)$ in Eq. (4.3) are considerably earlier than the observation times $t_{1}$ and $t_{2}$. Indeed, since $\left|\vec{x}_{1}-\vec{x}_{J}\left(s_{1}\right)\right| / c$ is about the time it takes a quasar ray to travel from Jupiter to Earth, $s_{1}$ in Eq. (4.2) corresponds to when the quasar ray passes near Jupiter. The same is true for $s_{2}$. Therefore, it is Jupiter's position at this moment that is revelant. This is physically reasonable since this is when the planet exerts its biggest influence on the rays.

Combining the results of the previous paragraphs, one sees that Eq. (4.1) agrees with Eqs. (2.3) and (3.9) since

$$
\begin{aligned}
& r_{1 J} \theta_{1}^{2}(s) \approx \xi_{1}^{2} / r_{1 J}, \\
& r_{2 J} \theta_{2}^{2}(s) \approx \xi_{2}^{2} / r_{2 J} .
\end{aligned}
$$

\section{The Faulty Analysis of the Jupiter/Quasar Experiment}

Since Eq. (4.1) is the leading order result in Eq. (2.7) up to unmeasurable corrections, and Eq. (2.7) has no $v_{J} / c$ dependence in it, the question arises as to how Fomalont and Kopeikin exacted $c_{g}$ from their data. Although the details of the analysis have not been revealed, I have surmised what transpired through their publications and through correspondence with Sergei Kopeikin.

Fomalont and Kopeikin express the result for the Shapiro delay time difference in Eq. (4.1) of

$$
S\left(s_{1}, s_{2}\right) \equiv \frac{2 G_{N} M_{J}}{c^{3}} \ln \left[\frac{r_{1 J}\left(s_{1}\right)+\vec{K} \cdot \vec{r}_{1 J}\left(s_{1}\right)}{r_{2 J}\left(s_{2}\right)+\vec{K} \cdot \vec{r}_{2 J}\left(s_{2}\right)}\right]
$$


which is a function of retarded times $s_{1}$ and $s_{2}$, as a fuction of the observation time $t_{1}$. The leading order contribution to $S\left(s_{1}, s_{2}\right)$ is

$$
\frac{4 G_{N} M_{J}}{c^{3}} \ln \left[\frac{\xi_{1}\left(s_{1}\right)}{\xi_{2}\left(s_{2}\right)}\right]
$$

If Jupiter is moving toward (or away from) the quasar then the distances $\xi_{1}(t)$ and $\xi_{2}(t)$ between Jupiter and quasar-ray-trajectories decrease (or increase) with time $t$ and are smaller (or larger) if evaluated at the time of observation:

$$
\begin{aligned}
& \xi_{1}\left(s_{1}\right) \approx \xi_{1}\left(t_{1}\right)+\vec{n} \cdot \vec{v}_{J} \frac{R_{E J}}{c}, \\
& \xi_{2}\left(s_{2}\right) \approx \xi_{2}\left(t_{1}\right)+\vec{n} \cdot \vec{v}_{J} \frac{R_{E J}}{c} .
\end{aligned}
$$

See Figure 2.

As is physically clear, the difference $\Delta \xi=\xi_{1}(t)-\xi_{2}(t)$ does not change with time as Eq. (5.3) shows. However, the leading order result in Eq. (5.2) now does since the substitution in Eq. (5.3) leads to

$$
\ln \left[\frac{\xi_{1}\left(s_{1}\right)}{\xi_{2}\left(s_{2}\right)}\right]=\ln \left[\frac{\xi_{1}\left(t_{1}\right)}{\xi_{2}\left(t_{1}\right)}\right]+\frac{\vec{n} \cdot \vec{v}_{J} R_{E J}}{c}\left(\frac{1}{\xi_{1}\left(t_{1}\right)}-\frac{1}{\xi_{2}\left(t_{1}\right)}\right) .
$$

The second term in Eq. (5.4) is

$$
\frac{\vec{n} \cdot \vec{v}_{J} R_{E J}}{c}\left(\frac{1}{\xi_{1}\left(t_{1}\right)}-\frac{1}{\xi_{2}\left(t_{1}\right)}\right) \approx-\frac{\Delta \xi\left(t_{1}\right)}{\xi^{2}\left(t_{1}\right)} \frac{\vec{n} \cdot \vec{v}_{J} R_{E J}}{c}=\frac{\vec{n} \cdot \vec{B}}{\theta_{1}^{2}\left(t_{1}\right)} \frac{\vec{n} \cdot \vec{v}_{J}}{c R_{E J}} .
$$

Summarizing,

$$
S\left(s_{1}, s_{2}\right)-S\left(t_{1}, t_{1}\right) \equiv \Delta_{R} \approx \frac{4 G_{N} M_{J} \vec{n} \cdot \vec{B} \vec{n} \cdot \vec{v}_{J}}{R_{E J} \theta_{1}^{2}\left(t_{1}\right) c^{4}} .
$$

The right-hand side of the equation contains the artificially $1 / \theta^{2}$ enhancement claimed by Kopeikin.[1, 12] It arises because the position of Jupiter changes as the quasar signals travel from the Jupiter region to Earth. The distance from Jupiter of the (almost) linear path of the quasar ray when it is detected at a VLBI station is different from the distance when it passed by Jupiter. Fomalont and Kopeikin used tables to determine Jupiter's position at $t_{1}$. Such information allows one to determine $S\left(t_{1}, t_{1}\right)$ 
analytically. So if one experimentally measures $S\left(s_{1}, s_{2}\right)$ and subtracts $S\left(t_{1}, t_{1}\right)$, then one can fit the data to $\Delta_{R}$ in Eq. (5.6) to extract the artificially generated second term.

Since $S\left(s_{1}, s_{2}\right)$ has no measureable $v_{J} / c$ dependence in it, there is implicit $v_{J} / c$ dependence in $S\left(t_{1}, t_{1}\right)$. This is because

$$
S\left(t_{1}, t_{1}\right) \approx-\frac{4 G_{N} M_{J} \vec{n} \cdot \vec{B}}{\theta_{1} r_{1 J} c^{3}}
$$

can be written as

$$
S\left(t_{1}, t_{1}\right) \approx-\frac{4 G_{N} M_{J} \vec{n} \cdot \vec{B}}{\left(\theta_{o b s}-\frac{\vec{n} \cdot \vec{v}_{J}}{c}\right) r_{1 J} c^{3}}
$$

to order $\left(v_{J} / c\right)^{2}$.

The Introduction argued that perhaps one way to extend Einstein's theory to the $c_{g} \neq c$ case is to replace $1 / c$ by $1 / c_{g}$ in the evalution of retarded times. Kopeikin does this in $S\left(s_{1}, s_{2}\right)$ when parametrizing it in terms of $t_{1}$. This, however, is incorrect. The difference $\Delta_{R}$ between $S\left(s_{1}, s_{2}\right)$ and $S\left(t_{1}, t_{1}\right)$ is due to Jupiter's change of position during the time it takes quasar rays to travel from the vicinity of Jupiter to Earth. This depends on the speed of rays, which is the speed of light, and not on the speed of gravity.

Thus, when data with error bars are fit to $\Delta_{R}$ and the fitting function uses $c_{g}$ instead of $c$, one is guaranteed to obtain the result $c_{g} \approx c$. Fomalont and Kopeikin's announcement that the speed of gravity is the speed of light to within $20 \%$ has no content.

\section{Can the Speed of Gravity Be Defined for the Jupiter/Quasar Exper- iment?}

This section addresses the theoretical issues raised in references $[7,8,9,10,11]$. Those references argued that Kopeikin's formula for the Shapiro time delay difference should involve the speed of light and not the speed of gravity. This debate is over how to define the speed of gravity in Einstein's general theory of relativity.

In the static frame, Jupiter is not moving. The curvature of space-time created by the massive planet is governed by Eq. (3.2) and is static. The effects of gravity are not propagating and the speed of gravity concept is non-existent. The velocity dependent corrections obtained in Eq. (3.9) arise due to the motion of the VLBI 
stations as they detect the radio waves. Since radio waves travel at the speed of light, the velocity dependent corrections must be proportional to $v_{J} / c$, and this is evident in the derivation of Eq. (3.9) in Section 3. It makes no sense to replace $v_{J} / c$ by $v_{J} / c_{g}$ from the viewpoint of the static frame.

On the other hand, in the frame in which Jupiter moves, the Introduction suggested that to extend Einstein's theory to the case $c_{g} \neq c$ one should replace retarded times as determined by $c$ by retarded times as determined by $c_{g}$. It would therefore seem as if the speed of gravity concept could be defined for this situation.

However, results as measured in one frame must be consistent with those measured in another. One is forced to conclude that the speed of gravity concept is theoretically inconsistent for the Jupiter/quasar experiment. If one tries to define the general theory of relativity with $c_{g} \neq c$ for this problem, one violates Galilean invariance.

Originally, C. Will has suggested that perhaps the speed of gravity might enter as a $v_{J}^{2} / c_{g}^{2}$ effect.[8] However, the above reasoning still applies. In relating the static frame results to the Jupiter-moving frame, one needs to use Lorentz transformations if higher velocity effects are to be considered. Therefore, an extension of Einstein's theory to describe the Jupiter/quasar experiment for which the linear velocity corrections are of the form $v_{J} / c$ but the quadratic corrections are of order $v_{J}^{2} / c_{g}^{2}$ for $c_{g} \neq c$ would violate Lorentz invarince (but not Galilean invariance). Recently, C. Will has also come to the same conclusion that $c_{g}$ does not appear in any higher power $\left(v_{j} / c\right)^{n}$ correction for a constantly moving Jupiter.[17]

As mentioned in my Physical Review Letter, the above argument would fail if Jupiter (or another massive object) were accelerating toward (or away from) the quasar rays (or other electromagnetic waves). It is possible that the speed of gravity could be defined for this situation. The parameter $c_{g}$ would then be associated with acceleration effects. It might be worth analyzing this case as a theoretical possibility. Unfortunately, it is unlikely that such a system within or beyond the solar system exists with sufficiently large effects as to be measurable with current VLBI instruments. 


\section{The Response by Fomalont and Kopeikin}

In fairness to Fomalont and Kopeikin, it should be said that they have not accepted the conclusions of my Physical Review Letter nor the criticisms of others. They have continued submitting papers $[13,18,19]$ arguing that they did indeed measure the speed of gravity. This section addresses those papers. Derivations of the formula in Eq. (4.1) mostly make up the content of these attempted rebuttals, while a few paragraphs are devoted to addressing the criticisms of other authors and of my Physical Review Letter. These paragraphs contain errors and false statements.

For example, reference [13] says, "This part of the experiment was drastically misunderstood by Samuel who assumed that we measured position of quasar with respect to Jupiter by measuring the relative position of the quasar with respect to Jupiter in radio." Similar statements appear in reference [19] ("A fundamental flaw in Samuel's interpretation was his assumption that the direction to Jupiter was directly measured by VLBI network in the detection experiment so he confused the propagation of gravity and the propagation of radio waves.") and reference [18] ("Unfortunately, Samuel incorrectly assumed that the experiment directly compared the radio position of the quasar with that of Jupiter, and that the direction of Jupiter was determined by a photon reflected from its surface."). My Physical Review Letter never made such statements, nor does this review: The criticism of the manner in which the data analysis was performed, which is presented in Section 5, focuses on the parametrization of and the expansion about the observation time $t_{1}$ by Kopeikin of the Shapiro time delay difference.

The unpublished work [18] continues "The experiment monitored the position of the quasar as a function of the atomic time by the arrival of the quasar's photons at the telescope, while the Jupiter's position was determined separately via a precise JPL ephemeris, evaluated at the same atomic time as the arrival of a photon (via standard transformations from ephemeris time to atomic time). Hence the actual angle used for measuring $\Delta$ is $\theta_{1}$, not Samuel's $\theta_{o b s}$. Thus, the $v_{J} / c$ correction $\Delta_{R}$ was clearly separated from $\Delta_{S}$ and measured with a precision of 20\%." Although these statements are intended to underpin my Physical Review Letter, anyone who 
truly understands the situation will realize that they actually support the Letter and the criticism in Section 5 levelled at the data analysis of the Fomalont/Kopeikin measurement.

Here is example of mistating the work of others as a means of defending the theory behind the quasar/Jupiter experiment. Reference [19] says, "Our definition of the speed of gravity is more general than that used by Asada, Samuel, and Will [[7], [14], [8]] who limited its meaning as the speed of propagation of gravitational waves." and "In their formulations of the experiment, these authors [Asada, Samuel, Will] assumed only far-field gravitational effects, where gravitational waves are dominant and differentiation between $c$ and $c_{g}$ occurs only at orders of $(v / c)^{2}$ beyond Shapiro delay and higher. This was one reason why the 'speed of light' was interpreted as causing the observed aberration. However, the experiment was performed in the near-field of the quasar radio wave-Jupiter interaction where gravitational modes not associated with gravitational waves are dominant."

The work of the three above-cited authors focused on the speed of propagation of the effects of gravity and not on the speed of gravity waves as falsely claimed in these quotes. My Physical Review Letter and this review did not discuss gravitational waves and have emphasized that the Shapiro time delay difference is due to relatively short-distance effects; I never "assumed only far-field gravitational effects". In actual fact, it is Kopeikin's formalism that ends up distorting and mixing up the short- and long-distance gravitational effects of Jupiter as explained in Section 5.

Reference [13] states, "The goal of the jovian deflection experiment was to distinguish two angles $\theta_{1}$ and $\theta_{o b s}$. Confirmation that the apparent position of the quasar in the sky makes the angle $\theta_{1}$ rather than $\theta_{o b s}$ with respect to Jupiter is a proof that gravity propagates with the speed $c_{g}$." This is not true: The difference between $\theta_{1}$ and $\theta_{o b s}$ is due to the motion of Jupiter during the period in which the quasar waves travel to Earth. Quasar waves travel at the speed of light so that $c_{g}$ cannot be extracted from a difference of $\theta_{1}$ and $\theta_{o b s}$; See Eqs. (1.4) and (5.6) where it is incorrect to replace $c$ by $c_{g}$. 
Reference [13] presents another example of faulty reasoning: "The equation

$$
\Delta\left(t_{1}, t_{2}\right)=-\frac{4 G_{N} M_{J}}{c^{3} \theta r_{1 J}}\left(\left(1+\frac{\vec{K} \cdot \vec{v}_{J}}{c_{g}}\right) \vec{n} \cdot \vec{B}+\vec{K} \cdot \vec{B}\left(\frac{\vec{n} \cdot \vec{v}_{E}}{c}-\frac{\vec{n} \cdot \vec{v}_{J}}{c_{g}}\right)\right)
$$

does not contain terms being quadratic in $1 / \theta$. It may make an impression that the orbital motion of Jupiter does not provide any significant deviation from the Einstein's prediction of the light deflection because all velocity-dependent terms in the right side of Eq. (7.1) are smaller than the main term (proportional to $(\vec{n} \cdot \vec{B}) / \theta$ ) by a factor of $10^{-4}$ and can not be observed with the present-day technology. This was the reason for Samuel's statement that terms of order $v / c$ beyond the Shapiro time delay are not observable. This statement is erroneous because the Shapiro time delay must be calculated in terms of the present position of Jupiter at the time of observation $t_{1}$."

The last statement is incorrect. Kopeikin's own formalism (the right-hand side of Eq. (4.1)) leads to the conclusion that the Shapiro time delay difference $\Delta$ should be computed at the retarded time, which is near the time at which the quasar rays pass Jupiter. Furthermore, it is not necessary to evaluate $\Delta$ at $t_{1}$ : Since the Shapiro time delay difference is generated when the rays are in the vicinity of Jupiter, $t_{1}$, which determines the retarded time $s_{1}$ in Eq. (4.2), may be evaluated at any time after the rays have passed well beyond Jupiter. As is physically clear, tens of millions of kilometers beyond Jupiter, the Shapiro time delay difference will have been almost $100 \%$ generated and then remain essentially unchanged. As is evident from Eqs. (2.3) and (3.10), there is only very weak dependence of $\Delta$ on the Earth-Jupiter distance $R_{E J}$.

In both references [13] and [19], Kopeikin claims that his formalism is Lorentz invariant, which is not the case. In Section 6 , we argued that if $c_{g} \neq c$ then not only is Lorentz invariance violated for the Jupiter/quasar experiment but also Galilean invariance.

Kopeikin derived Eq. (7.1) in part to show that his formalism agreed with the result in my Physical Review Letter when $c_{g}=c$. Eq. (7.1) is Kopeikin's generalization to arbitrary values of the speed of gravity. In this equation, $\vec{v}_{E}$ and $\vec{v}_{J}$ are the velocities of the Earth and Jupiter as determined in a coordinate system in which the Sun is at rest. 
However, Eq. (7.1) does not depend on $\vec{v}_{J}-\vec{v}_{E}$ as required by Galilean invariance. The frame-dependence is manifest: Hypothetically place the Sun at another location, give it a velocity of $\vec{v}_{S}$ relative to the original static Sun, have the Earth and Jupiter move with the same velocities that they originally had in the Jupiter/quasar experiment, then Eq. (7.1) becomes

$$
\begin{gathered}
\Delta\left(t_{1}, t_{2}\right)= \\
-\frac{4 G_{N} M_{J}}{c^{3} \theta r_{1 J}}\left(\left(1+\frac{\vec{K} \cdot\left(\vec{v}_{J}-\vec{v}_{S}\right)}{c_{g}}\right) \vec{n} \cdot \vec{B}+\vec{K} \cdot \vec{B}\left(\frac{\vec{n} \cdot\left(\vec{v}_{E}-\vec{v}_{S}\right)}{c}-\frac{\vec{n} \cdot\left(\vec{v}_{J}-\vec{v}_{S}\right)}{c_{g}}\right)\right)
\end{gathered}
$$

because, with respect to the "new" Sun, Jupiter and the Earth now move with velocities $\vec{v}_{J}-\vec{v}_{S}$ and $\vec{v}_{E}-\vec{v}_{S}$. The dependence on the "new" Sun's velocity $\vec{v}_{S}$ does not drop out. How can the Shapiro time delay difference due to Jupiter and observed on Earth be so dependent on the Sun's motion? The answer is that Eq. (7.1) is wrong. In a frame in which both the Earth and Jupiter move, the correct result is Eq. (3.9) with $\vec{v}_{J}$ replaced by $\vec{v}_{J}-\vec{v}_{E}$.

Reference [19] emphasizes incorrectly that it is the postion of Jupiter at the time of observation that is relevant: "the gravitational force, acting on photons as a space-like vector, is sensitive to the present position of Jupiter." For the sake of argument, let us assume that this is the case. When Kopeikin expands about the time of observation $t_{1}$, he obtains, for the case $c_{g}=c$, a $v_{J} / c$ correction of[1]

$$
\Delta\left(t_{1}, t_{2}\right)=-\frac{4 G_{N} M_{J}}{\theta_{1}^{2} r_{1 J} c^{4}}\left(\vec{B} \cdot \vec{v}_{J}-\vec{K} \cdot \vec{v}_{J} \vec{K} \cdot \vec{B}\right) .
$$

It is easy to see that this equation leads to physically unreasonable results. Suppose, for example, that Jupiter is moving toward the quasar rays. Hypothetically place a second planet twice as far away as Earth is to Jupiter and put VLBI stations on it along the same lines of observation as determined by quasar ray trajectories that pass through VLBI stations on Earth. Then one would expect to measure to a high precision the same Shapiro time delay difference. However, Eq. (7.3) predicts that more than twice the Earth-based correction will be measured. This follows because $1 /\left(\theta_{1}^{2} r_{1 J}\right)=r_{1 J} / \xi_{1}^{2}\left(t_{1}\right)$ where $\xi_{1}\left(t_{1}\right)$ is the distance between Jupiter and the worldline of the quasar ray 1 at the time of observation $t_{1}$. In making the observations 
"downstream" on the hypothetical planet, $r_{1 J}$ is doubled and $\xi_{1}\left(t_{1}\right)$ is smaller if Jupiter is moving toward the rays because the measurement is made later compared to the Earth-based one. How can Jupiter, which is so far away, have such a dramatic long-ranged influence on $\Delta$ ?

The answer is that $v_{J} / c$ corrections are not given by Eq. (7.3); The correct result is given in Eq. (3.9). Kopeikin should not have performed an expansion about the observation time that separated the leading Shapiro time difference result into two pieces. As explained in Section 5, his leading order result actually has implicit $v_{J} / c$ dependence in it (because it is written in terms of $\theta_{1}$ instead of $\theta_{\text {obs }}$ or $\xi$ ), which, when combined with his purported $v_{J} / c$ correction, gives the $v_{J} / c$-independent result of Eq. (2.7) to order $v_{J}^{2} / c^{2}$. This leading order term does not depend on how far "downstream" the measurement is made. The same is true of the $v_{J} / c$ correction in Eq. (3.9).

\section{Summary}

This work provides the leading $v_{J} / c$ corrections to the Shapiro time delay difference and shows that they do not correspond to the ones used in the data analysis by Fomalont and Kopeikin even when the speed of gravity parameter $c_{g}$ is set equal to $c$. The error made by Kopeikin is that he separated the leading $v_{J} / c$-independent term into two pieces by expanding about the time of observation $t_{1}$ through the subtraction procedure involving $\Delta_{R}$ in Eq. (5.6). This introduced an artificially $v_{J} / c$-dependent term. It is enhanced by $1 / \theta_{1}$ and is an artefact of the $t_{1}$ expansion. Although this $v_{J} / c$-dependent term should depend the speed of light $c$ because it is related to the change in position of Jupiter during the time in which radio waves travel to Earth, Kopeikin incorrectly replaces $c$ by $c_{g}$. Hence, when data is parametrized in this way, one is guaranteed to obtain a result for $c_{g}$ that is the speed of light to within experimental errors. Finally, when $c_{g} \neq c$ we agree with others $[7,9,10,11]$ that the theoretical formalism[16, 21, 12, 20, 13, 18, 19] of Kopeikin and his co-workers is flawed when applied to Jupiter/quasar system and demonstrated this by showing that not only does it violate Lorentz invariance but also Galilean invariance. While the Jupiter/quasar measurement was an extraordinary experimental undertaking and 
a fine achievement in precision, it had nothing to do with the determination of the speed of gravity.

\section{Acknowledgments}

I thank Professor Clifford Will for some useful comments and suggestions. This work is supported in part by the Director, Office of Science, Office of High Energy and Nuclear Physics, of the Department of Energy under contract number DE-AC0376SF00098. Correspondence with Sergei Kopeikin concerning many issues is acknowledged.

\section{References}

[1] S. M. Kopeikin, "Testing Relativistic Effect of Propagation of Gravity by VeryLong Baseline Interferometry", Astrophys. J. 556, L1 (2001) gr-qc/0105060.

[2] I. I. Shapiro, Phys. Rev. Lett. 13, 789 (1964).

[3] I. I. Shapiro et al., Phys. Rev. Lett. 20, 1265 (1968).

[4] I. I. Shapiro et al., Phys. Rev. Lett. 26, 1132 (1971).

[5] "Einstein Was Right on Gravity's Velocity", New York Times, January 8, 2003, Section A, p.13.

[6] E. Fomalont and S.M.Kopeikin, "The Measurement of the Light Deflection from Jupiter: Experimental Results", Astrophys. J. Lett. 598, 704 (2003), astroph/0302294.

[7] H. Asada, "The Light-cone Effect on the Shapiro Time Delay", Astrophys. J. Lett. 574, L69 (2002), astro-ph/0206266.

[8] C. M. Will, "Propagation of Speed of Gravity and Relativistic Time Delay", Astrophys. J. 590, 683 (2003). astro-ph/0301145.

[9] Joshua A. Faber, "The Speed of Gravity Has Not Been Measured from Time Delays", astro-ph/0303346 v1. 
[10] H. Asada, "Comments on 'Measuring the Gravity Speed by VLBI' ", Proc. of "Physical Cosmology", the XVth Rencontres de Blois, 15-20 June 2003, astro$\mathrm{ph} / 0308343$.

[11] S. Carlip, "Model-Dependence of Shapiro Time Delay and the 'Speed of Gravity/Speed of Light' Controversy", gr-qc/0403060 v3.

[12] S. M. Kopeikin, "Post-Newtonian Treatment of the VLBI Experiment on September 8, 2002", Phys. Lett. A312, 147 (2003), gr-qc/0212121.

[13] S. M. Kopeikin, "The Speed of Gravity in General Relativity and Theoretical Interpretation of the Jovian Deflection Experiment", Class. Quantum Grav. 21, 1 (2004), gr-qc/0310059.

[14] S. Samuel, "On the Speed of Gravity and the $v / c$ Corrections to the Shapiro Time Delay", Phys.Rev. Lett.90, 231101 (2003), astro-ph/0304006.

[15] S. Weinberg, 1972, Gravitation and Cosmology, (John Wiley \& Sons, New York, 1972), pp. 407-609.

[16] S. M. Kopeikin and G. Schäfer, "Lorentz Covariant Theory of Light Propagation in Gravitational Fields of Arbitrary-Moving Bodies", Phys. Rev. D60, 124002 (1999), gr-qc/9902030.

[17] C. M. Will, private communication.

[18] E. Fomalont and S. M. Kopeikin, "The Ultimate Speed of Gravity and v/c Correction to the Shapiro Time Delay", gr-qc/0310065 v2.

[19] E. Fomalont and S. M. Kopeikin, "Aberration and the Speed of Gravity in the Jovian Deflection Experiment", astro-ph/0311063 v3.

[20] E. Fomalont and S. M. Kopeikin, "The Measurement of the Light Deflection from Jupiter: Theoretical Interpretation", astro-ph/0302462 v1.

[21] E. Fomalont and S. M. Kopeikin, "General relativistic model for experimental measurement of the speed of propagation of gravity by VLBI", Proceedings of 
the 6th European VLBI Network Symposium, Ros, E., Porcas, R.W., Zensus, J.A. (eds.), June 25th - 28th, 2002, Bonn, Germany, pp. 49 - 52, gr-qc/0206022.

\section{Figure Captions}

Figure 1. The Expected Effect of the Speed of Gravity on the Gravitational Force Due to the Motion of Jupiter.

Jupiter, shown as an open circle, is assumed to be moving toward the electromagnetic wave. In the top figure, $c_{g}=\infty$ and it is the instantaneous position of Jupiter at time $t$, which is given by the shaded circle, that is relevant. In the middle figure, $c_{g}=c$ and the relevant distance is farther away: Since it takes time for the gravitational influence of Jupiter to propagate to the electromagnetic wave, it is Jupiter's position at an earlier time that is relevant. In the lower figure, $c_{g}<c$.

Figure 2. The Definitions of Various Quantities Relevant in the Jupiter/Quasar Experiment.

The diagram is not drawn to scale for reasons of clarity, and, in particular, angles are much larger than in the actual experiment. 
This figure "figure1.jpg" is available in "jpg" format from: http://arxiv.org/ps/astro-ph/0412401v1 
This figure "figure2.jpg" is available in "jpg" format from: http://arxiv.org/ps/astro-ph/0412401v1 